\title{
Avaliação da potencialidade de argilas da formação geológica Calumbi e Riachuelo em Sergipe para aplicação em revestimento cerâmico
}

\section{(Evaluation of the potenciality of clays from the Calumbi and Riachuelo geological formation in Sergipe, Brazil, for application in ceramic tiles)}

\author{
J. R. Goes ${ }^{1}$, T. F. Azevedo ${ }^{1}$, T. X. C. Dutra ${ }^{1}$, V. B. Santos ${ }^{1}$, J. B. Severo Junior ${ }^{2}$, L. S. Barreto ${ }^{1}$ \\ ${ }^{1}$ Laboratório de Tecnologia de Materiais, Núcleo de Ciências e Engenharia de Materiais \\ ${ }^{2}$ Departamento de Engenharia Química, Centro de Ciências Exatas e de Tecnologia \\ Universidade Federal de Sergipe, Av. Marechal Rondon s/n, S. Cristóvão, SE, Brasil 49100-000
}

Resumo

\begin{abstract}
As argilas são os materiais mais importantes do ponto de vista tecnológico para fabricação de materiais cerâmicos. A sua composição química e mineralógica influencia fortemente as propriedades físicas e químicas que se deseja obter no produto acabado de cerâmica. O objetivo desse trabalho foi avaliar a potencialidade de argilas da formação geológica Calumbi e Riachuelo do estado de Sergipe para aplicação em revestimento cerâmico. Foram coletadas amostras de 3 depósitos argilosos (A, B e C) e caracterizadas por ensaios de granulometria, plasticidade, difração de raios $\mathrm{X}$, análises termogravimétrica e diferencial, infravermelho e fluorescência de raios $\mathrm{X}$. As principais fases identificadas foram quartzo, caulinita, ilita, calcita e feldspatos, sendo que os depósitos B e C apresentaram elevados teores de calcita. Constatou-se que para as condições do processamento cerâmico adotadas as argilas dos depósitos A e B devidamente misturadas são adequadas para serem utilizadas na indústria de processamento de pisos cerâmicos, em especial para a obtenção da classificação do grupo cerâmico BIII.
\end{abstract}

Palavras-chave: argilas, caracterização, revestimento cerâmico, grupo de absorção.

\begin{abstract}
Clays are the most important materials from the technological point of view, for the production of ceramic materials. Its chemical and mineralogical composition strongly influences the physical and chemical properties to be obtained in the finished product of ceramic. The aim of this paper was to evaluate the potential of clays of the Calumbi and Riachuelo geological formation in Sergipe, Brazil, for the application in ceramic tiles. Samples were collected from 03 clay deposits (" $A$ ", " $B$ " and " $C$ ") and characterized by granulometryassays, plasticity, $X$-ray diffraction, and differential thermogravimetric analysis, infrared and $X$-ray fuorescence. The main identified phases were: quartz, kaolinite, illite, calcite and feldspar, and deposits " $B$ " and " $C$ " showed high levels of calcite. It was found that for ceramic processing conditions adopted in this paper, the properly mixed clay deposits of " $A$ " and " $B$ " are suitable for use in the processing industry ceramic tiles, particularly for obtaining of the classification of the absorption group "BIII".
\end{abstract}

Keywords: clay, characterization, ceramic tile, absorption group.

\section{INTRODUÇÃO}

As argilas são os materiais mais importantes do ponto de vista tecnológico para fabricação de materiais cerâmicos [1]. O comportamento das argilas utilizadas na fabricação de materiais cerâmicos é fortemente dependente da sua composição química e mineralógica que influenciam suas propriedades físicas e químicas, cujas propriedades são essenciais aos produtos acabados de cerâmica $[1,2]$.

As formulações típicas de placas cerâmicas para revestimento consistem principalmente de componentes como caulim ou caulinita, feldspatos e quartzo que, em proporções adequadas, contribuem individualmente no processo tecnológico e desempenho dos produtos finais. A presença da argila confere a plasticidade, os feldspatos atuam como agente fundente e o quartzo como material de enchimento $[3,4]$. Frações granulométricas (geralmente $<2 \mu \mathrm{m}$ ) ajudam a formação de plasticidade, que é um parâmetro necessário para a boa propriedade de moldagem de produção da cerâmica $[5,6]$. No processo de fabricação de placas cerâmicas, as matérias-primas são produzidas através de tecnologia de pó, seguidos normalmente por prensagem e queima $[7,8]$. Durante a etapa de queima, em temperaturas elevadas, uma série de transformações ocorre por interações entre os três componentes básicos (argila / feldspato / quartzo). As novas fases formadas são decisivas para as propriedades finais dos produtos cerâmicos $[7,9]$. Segundo a Associação Nacional dos Fabricantes de Cerâmica para Revestimentos, Louças Sanitárias e Congêneres (ANFACER) [10], o Brasil é o segundo produtor e o segundo maior consumidor mundial de placas cerâmicas para revestimentos. Ainda segundo a ANFACER, a região do nordeste do Brasil tem condições de se tornar um pólo cerâmico devido às suas condições favoráveis de matéria prima e disponibilidade de energia, 
Tabela I - Valores da taxa de absorção de água e do módulo de resistência à flexão para placas prensadas. [Table I - Values of water absorption rate and flexural strength modulus of pressed ceramic clay.

\begin{tabular}{ccccc}
\hline $\begin{array}{c}\text { Grupo de absorção } \\
\text { (placa prensada) }\end{array}$ & \multicolumn{2}{c}{ Absorção de água (\%) } & \multicolumn{2}{c}{ Módulo de resistência à flexão (MPa) } \\
Média & Individual & Média & Individual \\
\hline BIa & Abs $\leq 0,5 \%$ & $\leq 0,6 \%$ & $\geq 35 \mathrm{MPa}$ & $\geq 32 \mathrm{MPa}$ \\
BIb & $0,5 \%<\mathrm{Abs} \leq 3 \%$ & $\leq 3,3 \%$ & $\geq 30 \mathrm{MPa}$ & $\geq 27 \mathrm{MPa}$ \\
BIIa & $3 \%<\mathrm{Abs} \leq 6 \%$ & $\leq 6,5 \%$ & $\geq 22 \mathrm{MPa}$ & $\geq 20 \mathrm{MPa}$ \\
BIIb & $6 \%<\mathrm{Abs} \leq 10 \%$ & $\leq 11 \%$ & $\geq 18 \mathrm{MPa}$ & $\geq 16 \mathrm{MPa}$ \\
& & & $\geq 15 \mathrm{MPa}(\mathrm{e} \geq 7,5 \mathrm{~mm})$ \\
BIII & Abs $\geq 10 \%$ & $\geq 9 \%$ & & $\geq 12 \mathrm{MPa}(\mathrm{e} \leq 7,5 \mathrm{~mm})$ \\
\hline
\end{tabular}

Fonte: NBR 13817:1997 e 13818:1997 da ABNT

além de um mercado consumidor em desenvolvimento e de boa localização geográfica para exportação.

O estado de Sergipe, apesar de possuir as condições para se tornar um pólo cerâmico, contribuiu em 2011 com apenas $1,06 \%$ de toda produção brasileira. Estudos recentes foram feitos para investigar as causas do aparecimento de manchas após o uso de placas cerâmicas de revestimento [11], e sobre a caracterização química e mineralógica das argilas do estado de Sergipe utilizadas na produção de cerâmica vermelha [12]. A placa cerâmica fabricada no estado de Sergipe é processada por moagem por via seca e fabricada pelo método de prensagem, sendo classificada no grupo de absorção BIIb, de acordo com as normas técnica da Associação Brasileira de Normas Técnicas. A Tabela I apresenta para cada grupo de classificação, os respectivos valores e limites exigidos para absorção de água e módulo de resistência à flexão para placas prensadas.

O objetivo desse trabalho foi avaliar a potencialidade de argilas da formação geológica Calumbi e Riachuelo do estado de Sergipe para aplicação em revestimento cerâmico. Aspectos importantes das argilas, tais como análise granulométrica, limites de Atterberg, composição química, mineralógica e comportamento térmico foram investigados.

\section{MATERIAIS E MÉTODOS}

Foram coletadas amostras em 3 depósitos argilosos, identificados como depósitos A, B e C, localizados no estado de Sergipe. De acordo com o mapa geológico do estado de Sergipe (CODISE/CPRM - Serviço Geológico do Brasil, 1997), a identificação geológica da bacia sedimentar do depósito A pertence ao Grupo Piaçabuçu com formação Calumbi contendo argilito e folhelho, cinzentos a esverdeados, com intercalações de arenitos finos a grossos. Nesse depósito observou-se a presença de gipsita cristalizada no formato fibroso [12]. Os depósitos B e C pertencem ao Grupo Sergipe com Formação Riachuelo, sendo o depósito B, correspondente ao Membro Taquari, contendo calcilutito e folhelho, cinzentos interestratificados, e o depósito C, correspondente ao Membro Angico contendo arenitos brancos, finos a conglomeráticos, com intercalações de siltito, folhelho e calcáreo.
As amostras foram extraídas de diferentes localizações e profundidades, e identificadas como VR1,VR3 e VR5 (depósito A), PR1 e PR2 (depósito B), e DFR1 (depósito C). As amostras foram quarteadas, secas a $110{ }^{\circ} \mathrm{C}$ durante $24 \mathrm{~h}$, desagregadas manualmente em almofariz e pilão, e peneiradas em malha 200 mesh $(0,074 \mathrm{~mm})$, de acordo com a NM 27:2000 ABNT. A análise granulométrica foi realizada pelo método de peneiramento e sedimentação de acordo com a norma NBR 7181:1984 (ABNT). Os limites de Atterberg, limite de liquidez (LL) e limite de plasticidade (LP) foram obtidos de acordo com a NBR 6459:84 e a NBR 7180:84 (ABNT), respectivamente. O índice de plasticidade (IP) foi encontrado pela diferença entre os dois limites LL e LP.

O comportamento da composição e cristalização das argilas foi analisado por termogravimetria e análise diferencial (TGA/DTA, SDT 2960, TA Instr.), a uma taxa de aquecimento de $10{ }^{\circ} \mathrm{C} / \mathrm{min}$ desde a temperatura ambiente até $1200{ }^{\circ} \mathrm{C}$, em atmosfera de $\mathrm{N}_{2}$. As fases das argilas foram identificadas por análise de difração de raios $\mathrm{X}$ em um equipamento Rigaku com radiação $\operatorname{Cuk}_{\alpha}(\lambda=1,5148 \mathrm{~A})$, velocidadede $5^{\circ} / \mathrm{min}$ para $2 \theta$ de $5^{\circ}$ a $80^{\circ}$. Os padrões de difração de raios $\mathrm{X}$ foram identificados por meio do banco de dados JCPDS.

A determinação da composição química foi obtida através da análise de energia dispersiva de raios X (EDX720, Shimadzu). Os espectros de infravermelho por transformada de Fourier (FTIR) foram obtidos no espectrofotômetro 640-IR-T-IR com varredura de 4000 a $600 \mathrm{~cm}^{-1}$.

Após quarteamento e secagem, as amostras foram desagregadas manualmente em almofariz e pilão, e peneiradas em malha 48 mesh, misturadas, granuladas com teor de $8 \%$ de umidade (umidade utilizada pela indústria sergipana estudada), prensadas a $32,7 \mathrm{MPa}$ (obtendo-se a placa verde), secas em estufa a $110^{\circ} \mathrm{C}$ por $24 \mathrm{~h}$ (obtendo-se a placa seca) e submetidas a tratamento térmico (obtendo-se a placa queimada). Para a prensagem, foi utilizada uma prensa manual uniaxial com capacidade de 15 ton. O molde foi confeccionado em aço SAE 1045, com a confecção de corpos de prova retangulares nas dimensões $60 \mathrm{~mm}$ x $20 \mathrm{~mm}$ x $5 \mathrm{~mm}$ [13].

Corpos de prova em duplicata foram preparados 
Tabela II - Formulação das misturas utilizadas para preparação dos corpos de provas.

[Table II - Formulation of the mixtures used for preparation of the test specimens.]

\begin{tabular}{ccccccc}
\hline \multirow{2}{*}{ Amostras } & \multicolumn{6}{c}{ Misturas } \\
& M1 & M2 & M3 & M4 & M5 & M6 \\
\hline VR1 & $50 \%$ & $60 \%$ & $70 \%$ & $80 \%$ & $90 \%$ & $100 \%$ \\
PR2 & $50 \%$ & $40 \%$ & $30 \%$ & $20 \%$ & $10 \%$ & $0 \%$ \\
\hline
\end{tabular}

utilizando-se formulações com misturas, proporcionalmente em peso, das amostras VR1 e PR2 conforme Tabela II, para serem ensaiados com o objetivo de se determinar: a retração linear de acordo com a MBR 305 da ABNT; a taxa de absorção de água de acordo com a NBR 13818:97 da ABNT; a porosidade aparente de acordo com a norma americana C378-88 da ASTM; e a massa específica aparente de acordo com a norma americana C378-88 da ASTM.

A etapa de queima foi executada em um forno mufla com taxa de aquecimento $10{ }^{\circ} \mathrm{C} / \mathrm{min}$ até $1100{ }^{\circ} \mathrm{C}$ (temperatura próxima da utilizada pela indústria objeto deste estudo), com tempo de patamar de $30 \mathrm{~min}$ e resfriamento natural no próprio forno.

\section{RESULTADOS E DISCUSSÃO}

A distribuição do tamanho das partículas e os limites de Atterberg das amostras estudadas estão listados na Tabela III. Os resultados mostram que as amostras analisadas têm fração de argila $(\phi<2 \mu \mathrm{m})$ de 23 a $50 \%$, proporção de silte $(60 \mu \mathrm{m}<\phi<2 \mu \mathrm{m})$ de 45 a $73 \%$, e a fração de areia $(\phi>60$ $\mu \mathrm{m})$ de 3 a $6 \%$. Observa-se que as amostras VR1, VR2 e VR3 contêm alto teor de argila em comparação com as amostras PR1, PR2 e DFR1. Para o processamento de produtos de cerâmica, atenção especial deve ser dada à fração mais fina $(\phi<2 \mu \mathrm{m})[14]$.

Os limites de plasticidade e liquidez (LP e LL) das amostras variam entre 23 e $30 \%$ e 32 e $63 \%$, respectivamente. Comparando as amostras, a argila VR1 apresentou os maiores valores de LL, LP e IP. As diferenças no IP (8-34\%) das amostras estão relacionadas com os dados da distribuição de tamanhos de partículas. Os valores de índice de plasticidade aumentam juntamente com o aumento do teor de argila $(\phi$ $<2 \mu \mathrm{m}$ ), produzindo alta plasticidade para as amostras de depósito "A". Assim, a fração do tamanho das partículas ( $\phi$ $<2 \mu \mathrm{m})$ pode afetar os limites de Atterberg $([5,18,19])$. Os valores típicos do índice de plasticidade de Atterberg para argila caulinítica variam de $5 \%$ a $22 \%$, para argila ilita de $39 \%$ a $51 \%$, e para argilas montmorilonita, até $600 \%$ [20]. Com base nos resultados dos IP obtidos, as amostras das argilas coletadas são características de argilas caulinitas.

Muitas das propriedades finais dos produtos cerâmicos são afetadas diretamente pelo tamanho dos grãos das matérias primas [15]. Uma das ferramentas utilizadas para orientar no estudo da composição da granulometria de massas cerâmicas é o diagrama de Winkler, que permite predizer sua recomendação para fabricação de determinados tipos de produtos de cerâmica vermelha, assim como possibilita proceder aos ajustes necessários de uma composição granulométrica dosando-se adequadamente grãos finos, médios e grossos [16, 17]. A Fig. 1 apresenta a posição das amostras VR1, VR3, VR5, PR1, PR2 e DFR1 no referido diagrama. As argilas VR3 e VR5 do depósito "A", são as mais indicadas para produção de placas cerâmicas, e que a argila VR1 do mesmo depósito necessita de um aumento do percentual da fração de não finos $(\phi>20 \mu \mathrm{m})$, que pode ser obtido misturando-se adequadamente com as argilas PR1 do depósito "B". Com base no diagrama de Winkler foram preparadas as formulações constantes na Tabela II, que apresenta os respectivos percentuais em peso de cada amostra utilizados para preparação dos corpos de provas.

A Fig. 2 apresenta a posição das formulações M1, M2, M3, M4, M5 e M6 no diagrama de Winkler. Ocorreu uma melhoria da composição granulométrica em relação à indicação do produto cerâmico indicado (tipo I) do diagrama de Winkler. Observa-se ainda a necessidade de se ter um aumento de 5 a $10 \%$ no percentual de não finos $(\phi>20 \mu \mathrm{m})$ para o ajuste granulométrico do referido diagrama.

A Tabela IV lista as composições químicas das amostras

Tabela III - Características físicas das amostras das argilas. [Table III - Physical characteristics of the clay samples.]

\begin{tabular}{|c|c|c|c|c|c|c|c|}
\hline \multirow[b]{2}{*}{ Depósitos } & \multirow[b]{2}{*}{ Amostras } & \multicolumn{3}{|c|}{ Distribuição de tamanho de partícula (\%) } & \multicolumn{3}{|c|}{ Limites de Atterberg (\%) } \\
\hline & & $\begin{array}{c}\text { Areia } \\
(\phi>60 \mu \mathrm{m})\end{array}$ & $\begin{array}{c}\text { Silte } \\
(60 \mu \mathrm{m}>\phi>2 \mu \mathrm{m})\end{array}$ & $\begin{array}{c}\text { Argila } \\
(\phi<2 \mu \mathrm{m})\end{array}$ & LL & LP & IP \\
\hline \multirow{3}{*}{ A } & VR1 & 3,79 & 45,95 & 50,26 & 63,00 & 29,84 & 33,16 \\
\hline & VR3 & 5,42 & 50,92 & 43,66 & 42,50 & 24,36 & 18,14 \\
\hline & VR5 & 4,59 & 48,73 & 46,68 & 50,00 & 28,21 & 21,79 \\
\hline \multirow{2}{*}{ B } & PR1 & 5,85 & 70,86 & 23,30 & 34,30 & 25,26 & 9,04 \\
\hline & PR2 & 3,39 & 65,25 & 31,36 & 35,60 & 25,56 & 10,04 \\
\hline $\mathrm{C}$ & DFR1 & 4,64 & 72,28 & 23,08 & 32,00 & 23,07 & 8,93 \\
\hline
\end{tabular}

$L L=$ Limite de Liquidez; $L P=$ Limite de Plasticidade; $I P=$ Índice de Plasticidade. 
das argilas naturais expressas na forma de óxidos. Ela mostra que a maioria dos óxidos presentes são $\mathrm{SiO}_{2}, \mathrm{Al}_{2} \mathrm{O}_{3}$ e $\mathrm{FeO}_{3}$,

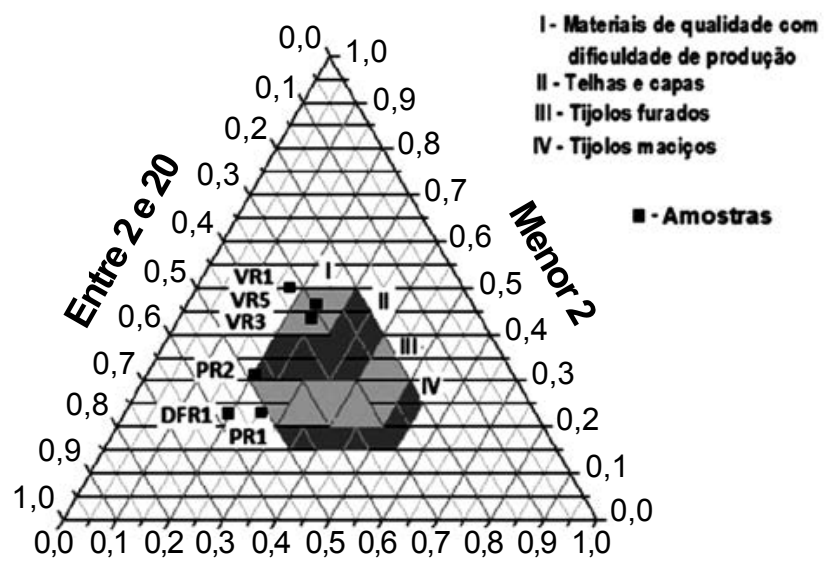

\section{Maior 20}

Figura 1: Posições das amostras VR1, VR3, VR5, PR1, PR2 e DFR1 no diagrama granulométrico de Winkler.

[Figure 1: Position of the samples VR1,VR3, VR5, PR1, PR2 and DFR1 in the Winkler granulometric diagram.]

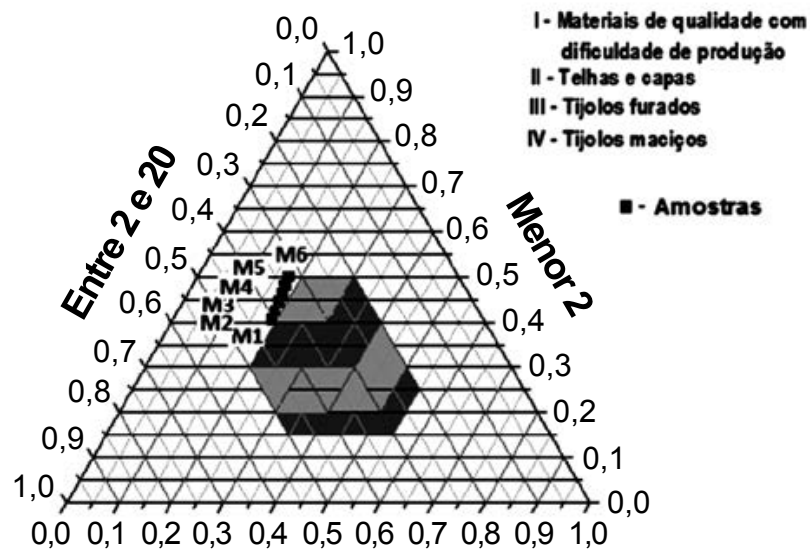

\section{Maior 20}

Figura 2: Posições das formulações M1, M2, M3 M4, M5 e M6 no diagrama granulométrico de Winkler.

[Figure 2: Position of the formulations M1, M2, M3, M4, M5 andM6inthe Winkler granulometric diagram.]
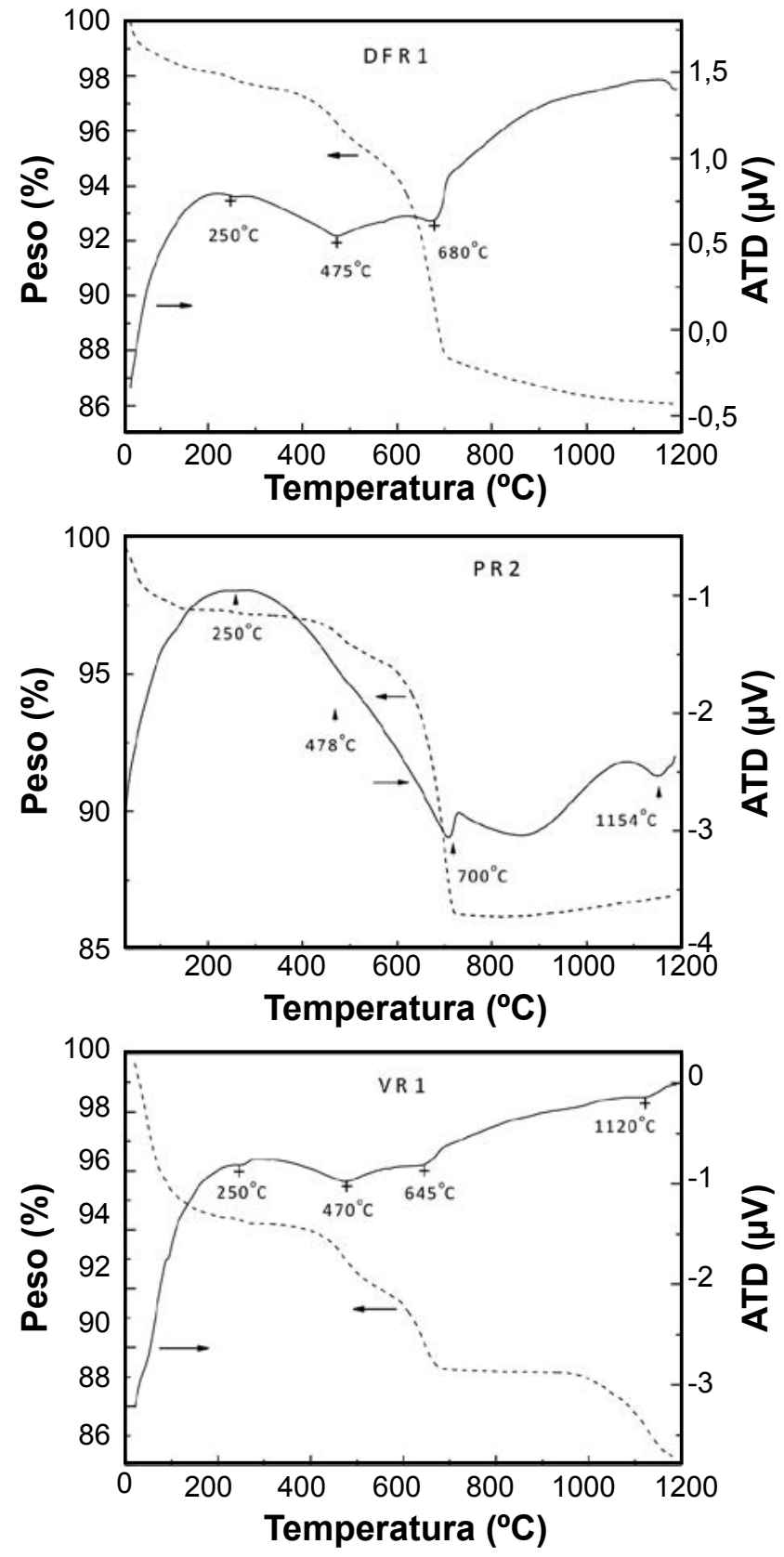

Figura 3: Curvas TGA/DTA das amostras VR1, PR2 e DFR1. [Figure 3: TGA/DTA curves of the samples VR1, PR2 and DFR1.]

Tabela IV - Composição química das amostras das argilas.

[Table IV - Chemical composition of the clay samples.]

Composição (\% em peso)

Depósitos Amostras

$\begin{array}{llllllllll}\mathrm{SiO}_{2} & \mathrm{Al}_{2} \mathrm{O}_{3} & \mathrm{Fe}_{2} \mathrm{O}_{3} & \mathrm{CaO} & \mathrm{K}_{2} \mathrm{O} & \mathrm{Na}_{2} \mathrm{O} & \mathrm{MgO} & \mathrm{P}_{2} \mathrm{O}_{5} & \mathrm{TiO}_{2} & \text { Outros }\end{array}$

\begin{tabular}{|c|c|c|c|c|c|c|c|c|c|c|c|}
\hline \multirow{3}{*}{ A } & VR1 & 45,94 & 11,40 & 4,11 & 5,15 & 2,01 & 0,54 & 1,42 & 0,22 & 0,53 & 28,70 \\
\hline & VR3 & 54,40 & 11,90 & 4,26 & 3,48 & 2,50 & 0,70 & 1,69 & 0,19 & 0,56 & 20,30 \\
\hline & VR5 & 56,00 & 13,99 & 4,71 & 1,07 & 2,65 & 0,69 & 1,76 & 0,13 & 0,65 & 18,30 \\
\hline \multirow{2}{*}{ B } & PR1 & 49,60 & 12,20 & 4,08 & 10,50 & 2,43 & 0,69 & 1,65 & 0,20 & 0,62 & 17,97 \\
\hline & PR2 & 50,10 & 12,60 & 4,25 & 7,56 & 2,36 & 0,72 & 1,79 & 0,18 & 0,63 & 19,80 \\
\hline $\mathrm{C}$ & DFR1 & 51,40 & 13,60 & 4,77 & 7,18 & 2,53 & 0,79 & 1,54 & 0,17 & 0,70 & 17,30 \\
\hline
\end{tabular}


característicos das argilas utilizadas nas indústrias cerâmicas $[11,14,21]$. A principal diferença entre as amostras dos depósitos $\mathrm{B}$ e $\mathrm{C}$ em relação ao depósito $\mathrm{A}$ é um menor teor de $\mathrm{CaO}$ em A. É bem conhecido que os conteúdos elevados de carbonato de cálcio podem proporcionar porosidade nos corpos cerâmicos durante a queima, justificando a elevada
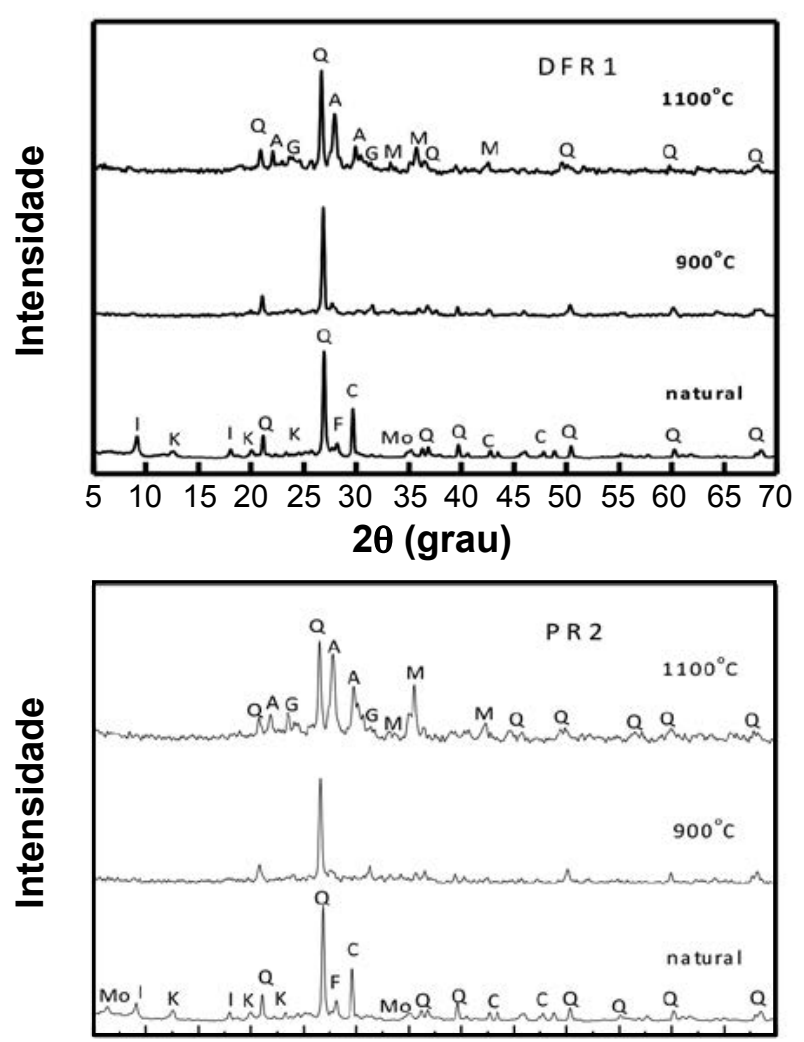

$\begin{array}{llllllllllllll}5 & 10 & 15 & 20 & 25 & 30 & 35 & 40 & 45 & 50 & 55 & 60 & 65 & 70\end{array}$ $2 \theta$ (grau)

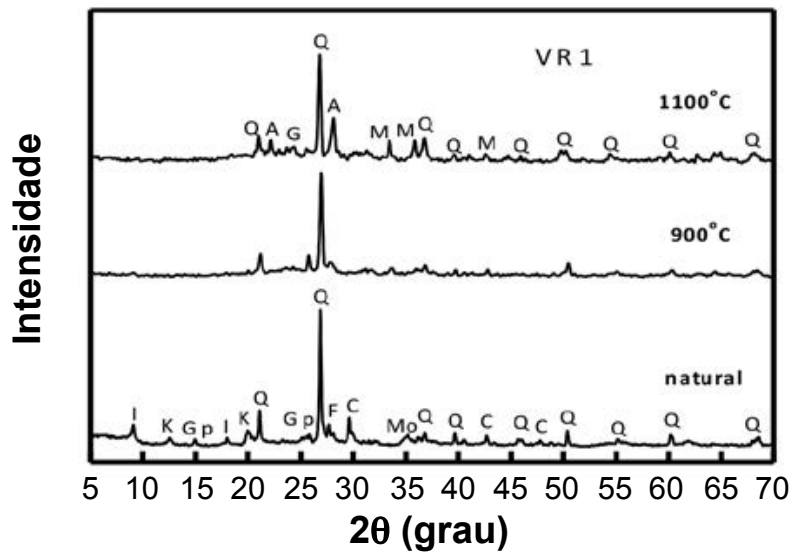

Figura 4: DRX das amostras VR1, PR2 e DFR1 naturais e calcinadas a $900{ }^{\circ} \mathrm{C}$ e a $1100{ }^{\circ} \mathrm{C}$. Q - quartzo; C - calcita; I - ilita; K - caulinita; Gp - gipsita; Mo - montmorilonita; F - feldspatos; $\mathrm{Mu}$ - mulita; $\mathrm{G}$ - guelenita; A - anortita.

[Figure 4: XRD of the samples VR1, PR2 DFR1 natural and calcined at $900{ }^{\circ} \mathrm{C}$ and $1100{ }^{\circ} \mathrm{C} . Q$ - quartz, C - calcite, I-illite, $K$ - kaolinite; $G p$ - gipsite, $M o$ - montmorillonite, $F$ - feldspar; $M u$ - mullite; $G$ - gehlenite; $A$ - anorthite.] perda de massa conforme observado nas análises de TGA/ DTA (Fig. 3).

As curvas TGA/DTA das amostras das argilas em estudo estão ilustradas na Fig. 3. Foram observados os seguintes picos endotérmicos: a aproximadamente $250^{\circ} \mathrm{C}$, relacionado à decomposição dos hidróxidos; a aproximadamente 470 ${ }^{\circ} \mathrm{C}$, devido a desidroxilação e remoção da matéria orgânica e, entre $645{ }^{\circ} \mathrm{C}$ e $700{ }^{\circ} \mathrm{C}$, relacionado à decomposição da calcita [13]. Nesta última região de temperatura um efeito endotérmico forte é observado em PR2 e DFR1. Para o primeiro pico endotérmico $\left(\sim 250{ }^{\circ} \mathrm{C}\right)$, a perda de massa de VR1, PR2 e DFR1 foi de 5,7\%, 2,7\% e 2,0\%, respectivamente. No pico aproximadamente a $470{ }^{\circ} \mathrm{C}$ há uma perda de massa de $6,9 \%, 3,6 \%$ e $3,8 \%$ para VR1, PR2 e DFR1, respectivamente. Observa-se que para estes dois primeiros picos endotérmicos VR1 apresenta perda de massa duas vezes maior em comparação com PR2 e DFR1. A perda de massa atribuída à decomposição da calcita foi estimada em 2,5\% para VR1, 7,2\% para PR2 e 9,7\% para DFR1. Estes valores elevados confirmam maior conteúdo de calcita para as argilas PR2 e DFR1. Finalmente, um pequeno pico endotérmico a $1120{ }^{\circ} \mathrm{C}$ e $1150{ }^{\circ} \mathrm{C}$ para VR1 e PR2, respectivamente, pode ser relacionado com a nucleação de mulita [13], como também pode ser observado conforme indicado pelos padrões do DRX das argilas submetidas a tratamento térmico. A perda de massa total das argilas nos ensaios TG/DTA ficou em $\sim 14 \%$.

A Fig. 4 mostra os resultados de difração de raios $\mathrm{X}$ de argilas naturais, e após a calcinação a $900{ }^{\circ} \mathrm{C} \mathrm{e} 1100^{\circ} \mathrm{C}$. Todas as argilas apresentaram as fases cristalinas características da caulinita, ilita, montmorilonita, quartzo, gibsita, calcita e feldspato $[11,22,23]$. Ficou bastante evidente o aumento da reflexão da calcita para as amostras PR2 e DFR1, em bom acordo com os resultados observados por TGA (Fig.3). Após calcinação a intensidade das reflexões de caulinita, ilita, montmorilonita, quartzo, gibsita e feldspato desapareceram, enquanto novas fases são formadas. Em $1100^{\circ} \mathrm{C}$, a mulita é identificada por DRX, em todas as amostras, como resultado da transformação térmica da ilita e da caulinita $[24,25]$.

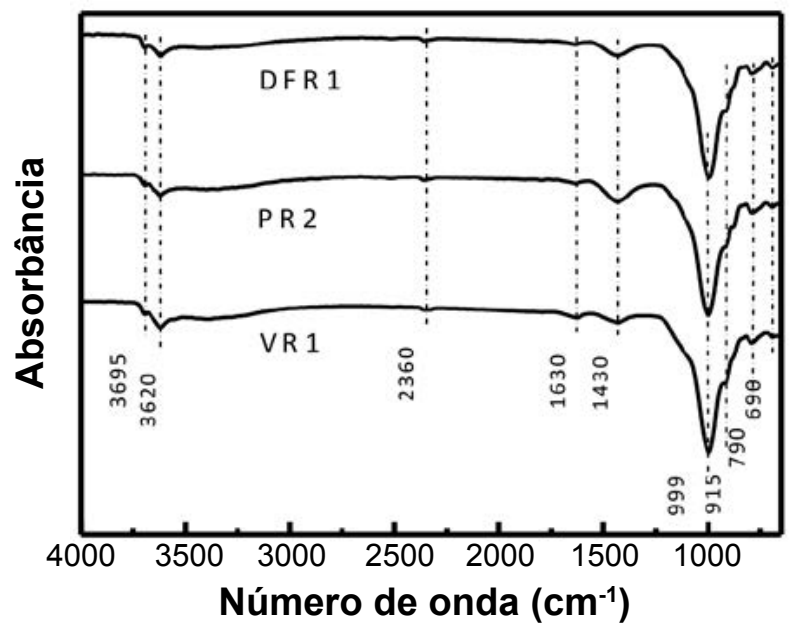

Figura 5: Espectros FTIR das amostras das VR1, PR2 e DFR1. [Figure 5: FTIR spectra of the samples VR1, PR2 and DFR1.] 
Tabela V - Resultados obtidos para as formulações M1, M2, M3, M4, M5 e M6. [Table V-Results for the formulations M1, M2, M3, M4, M5 and M6.]

\begin{tabular}{cccccc}
\hline Misturas & $\begin{array}{c}\text { Retração linear } \\
(\%)\end{array}$ & $\begin{array}{c}\text { Absorção } \\
\text { de água } \\
(\%)\end{array}$ & $\begin{array}{c}\text { Porosidade } \\
(\%)\end{array}$ & $\begin{array}{c}\text { Módulo de } \\
\text { resistência } \\
\text { à flexão } \\
(\mathrm{MPa})\end{array}$ & $\begin{array}{c}\text { Massa } \\
\text { específica aparente } \\
\left(\mathrm{g} / \mathrm{cm}^{3}\right)\end{array}$ \\
\hline \multirow{2}{*}{ M1 } & $-0,149$ & 13,065 & 24,712 & 14,02 & 1,891 \\
& $-0,050$ & 13,022 & 24,664 & 12,01 & 1,894 \\
M2 & 0,116 & 12,086 & 23,152 & 11,53 & 1,916 \\
& 0,149 & 12,212 & 23,252 & 11,34 & 1,904 \\
M3 & 0,298 & 11,583 & 22,301 & 11,62 & 1,925 \\
& 0,215 & 11,542 & 22,264 & 11,11 & 1,929 \\
M4 & 0,314 & 11,639 & 22,527 & 10,21 & 1,935 \\
& 0,413 & 11,014 & 21,392 & 11,34 & 1,942 \\
M5 & 0,612 & 10,048 & 19,845 & 12,86 & 1,975 \\
& 0,579 & 9,447 & 18,803 & 17,16 & 1,991 \\
M6 & 0,844 & 9,117 & 18,250 & 11,96 & 2,002 \\
& 0,910 & 9,013 & 18,037 & 11,76 & 2,001 \\
\hline
\end{tabular}

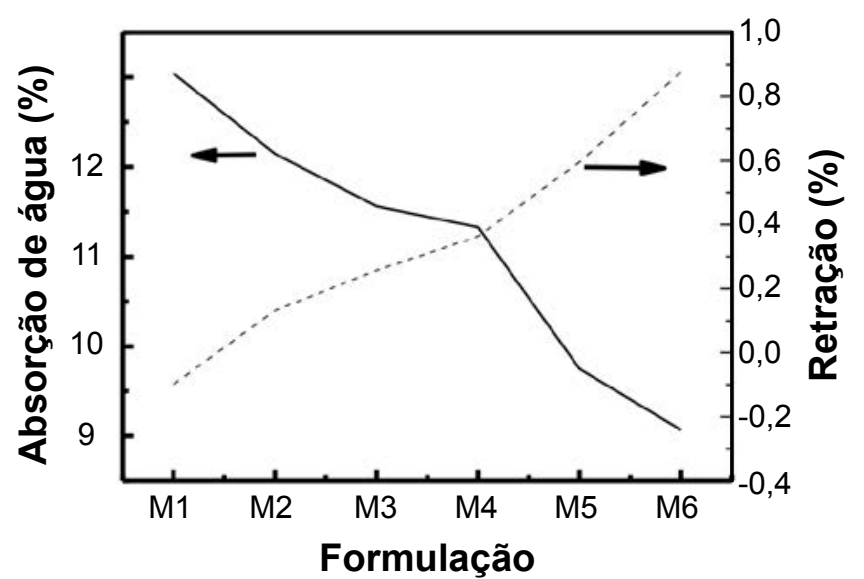

Figura 6: Variação da absorção de água e retração linear para as formulações M1, M2, M3, M4, M5 e M6.

[Figure 6: Variation of water absorption and linear shrinkage for formulations M1, M2, M3, M4, M5 and M6.]

Também pode ser visto que as fases típicas da guelenita $\left(\mathrm{Ca}_{2} \mathrm{Al}_{2} \mathrm{SiO}_{7}\right)$ e anortita $\left(\mathrm{CaAl}_{2} \mathrm{Si}_{2} \mathrm{O}_{8}\right)$ foram formadas durante o tratamento térmico, devido ao maior teor de cálcio nessas amostras $[9,26]$. Acima de $900^{\circ} \mathrm{C}$, os picos de calcita estavam ausentes, confirmando a sua decomposição. Assim, as placas cerâmicas preparadas com essa argila devem apresentar alta porosidade quando calcinadas em temperaturas acima de $900{ }^{\circ} \mathrm{C}$.

Os espectros de FTIR das amostras das argilas são mostrados na Fig. 5. Vê-se claramente que todas as amostras apresentam características semelhantes. A banda a cerca de $3700 \mathrm{~cm}^{-1}$ e $3620 \mathrm{~cm}^{-1}$ são características de alongamento de vibrações de $\mathrm{O}-\mathrm{H}$. O pico em $1630 \mathrm{~cm}^{-1}$ é devido à água de hidratação na superfície da argila [27, 28]. A banda de absorção em $1430 \mathrm{~cm}^{-1}$ está relacionada com a vibração da calcita. Bandas devido às vibrações de alongamento $\mathrm{SiO}_{2}$ são vistas em $999 \mathrm{~cm}^{-1}$, enquanto que a $915 \mathrm{~cm}^{-1}$ sugere a deformação Al-OH-Al [27, 29]. Picos de quartzo foram detectados próximo $790 \mathrm{~cm}^{-1}$ e $690 \mathrm{~cm}^{-1}$ [27, 28]. $\mathrm{O}$ pico a cerca de $2360 \mathrm{~cm}^{-1}$ pode ser atribuído ao $\mathrm{CO}_{2}$ atmosférico [30]. Assim, os resultados de FTIR confirmam adicionalmente os resultados dos ensaios de EDX, TGA/ DTA e DRX das amostras coletadas.

A Tabela $\mathrm{V}$ apresenta os resultados obtidos para as formulações estudadas para retração linear, absorção de água, porosidade, módulo de resistência à flexão e massa específica aparente, e a Fig. 6 apresenta graficamente o comportamento da absorção de água e da retração linear com a variação da proporção na mistura das matérias primas dos depósitos "A" e "B". Como pode ser observado para os corpos de provas estudados, à medida que se aumenta o percentual relativo à matéria prima do depósito A ocorre uma redução acentuada da taxa de absorção de água acompanhada de um grande aumento da retração linear. Foram observados pequenos desvios nas retitudes dos lados e empenos nas planicidades das placas preparadas com as formulações M5 e M6, provocadas provavelmente pela ocorrência de maior retração e a sua não uniformidade ao longo das dimensões da placa.

\section{CONCLUSÕES}

As amostras do depósito A apresentaram um alto teor de argila em comparação com as amostras dos depósitos $\mathrm{B}$ e $\mathrm{C}$, assim como maiores índices de plasticidade, o que exigirá uma especial atenção no processamento de produtos de cerâmica. A análise química revela que todas as argilas contêm predominantemente $\mathrm{SiO}_{2}, \mathrm{Al}_{2} \mathrm{O}_{3}$ e $\mathrm{FeO}_{3}$, acompanhados por um teor significativo de $\mathrm{CaO}$ (exceto 
amostras do depósito A). Isto explica a perda de massa elevada, devido à decomposição da calcita, para as amostras PR2 (depósito B) e DFR1 (depósito C). As amostras coletadas são características de argilas caulinitas, com presença de fases características da ilita, gibsita, quartzo, calcita e feldspato, enquanto que as fases mulita, guelenita e anortita foram observadas a $1100^{\circ} \mathrm{C}$. Estas novas fases são formadas principalmente por transformação térmica e uma série de reações da calcita com os argilominerais. A decomposição da calcita foi observada para todas as amostras nas temperaturas acima de $900{ }^{\circ} \mathrm{C}$. A presença de minerais de quartzo e calcita foi ainda confirmada por análise de FTIR. O elevado teor de calcita nos depósitos $\mathrm{B}$ e $\mathrm{C}$ retarda o processo de sinterização, causando maior porosidade ao revestimento, o que ocorre em menor proporção com as argilas do depósito A. Para as condições do processamento cerâmico adotado e nos valores de absorção de água, conforme normas técnicas 13817:1997 e 13818:1997 da ABNT, as placas obtidas com as formulações M5 e M6 podem ser classificadas no grupo de absorção BIIb, e as demais formulações classificadas no grupo de absorção BIII. Entretanto, há necessidade de melhorar os resultados do módulo de resistência à flexão em todas as formulações, e de redução da retração linear para as formulações M5 e M6.

\section{AGRADECIMENTOS}

Os autores agradecem o apoio financeiro da CAPES e do CNPq, e ao ITPS-Instituto de Tecnologias e Pesquisa do Estado de Sergipe, Brasil, pelas análises químicas.

\section{REFERÊNCIAS}

[1] H. Celik, Appl. Clay Sci. 50, 2 (2010) 245.

[2] S. Ferrari, A. F. Gualtieri, Appl. Clay Sci. 32, 1-2 (2006) 73.

[3] E. Kamseu, C. Leonelli, D. N. Boccaccini, P. Veronesi, P. Miselli, G. Pellacani, U. C. Melo, Ceram. Int. 33, 5 (2007) 851.

[4] J. Martín-Márquez, J. Ma. Rincón, M. Romero, J. Eur. Ceram. Soc. 30, 7 (2010) 1599.

[5] C. O. Modesto, A. M. Bernardin, Appl. Clay Sci. 40, 1-4 (2008) 15.

[6] A De Noni Jr., D. Hotza, V. C. Soler, E. S. Vilches, Mater. Sci. Eng. A527, 7-8 (2010) 1730.

[7] A. J. Souza, B. C. A. Pinheiro, J. N. F. Holanda, J. Mater. Proc. Technol. 210, 14 (2010) 1898.

[8] E. Sánchez, J. García-Ten, V. Sanz, A. Moreno, Ceram. Int. 36, 3 (2010) 831.
[9] M. M. Jordan, J. D. Martín-Martín, T. Sanfeliu, D. Gómez-Gras, C. de la Fuente, Appl. Clay Sci. 44, 1-2 (2009) 173.

[10] ANFACER - Associação Nacional dos Fabricantes de Cerâmica para Revestimentos, Louças Sanitárias e Congêneres, disponível em http://www.anfacer.org.br, acesso em 15/10/2013.

[11] A. C. S. Alcântara, M. S. S. Beltrão, H. A. Oliveira, I. F. Gimenez, L. S. Barreto, Appl. Clay Sci. 39, 3-4 (2008) 160.

[12] C. M. de O. Prado, Diss. Mestrado, Universidade Federal de Sergipe, S. Cristovão, SE (2011).

[13] P. Souza Santos, "Ciência e Tecnologia das Argilas", $2^{\text {a }}$ Ed., Vol. 1, Edgard Blücher, S. Paulo, SP (1989) 176.

[14] S. Mahmoudi, E. Srasra, F. Zargouni, Appl. Clay Sci. 42, 1-2 (2008) 125.

[15] M. Dondi, B. Fabbri, G. Guarini, Clay Minerals 33 (1998) 435.

[16] S. Pracidelli, F. G. Melchiades, Cerâmica Ind. 2, 1-2 (1997) 31.

[17] C. M. F. Vieira, S. N. Monteiro, J. Duailibi Fh., Ceram. Ind. 10, 1 (2005) 23.

[18] B. K. Ngun, H. Mohamad, S. K. Sulaiman, K. Okada, Z. A. Ahmad, Appl. Clay Sci. 53, 1 (2011) 33.

[19] F. A. de Andrade, H. Ali Al-Qureshi, D. Hotza, Mater. Res. 13, 3 (2010) 395.

[20] A. Barba, V. Beltrán, C. Felíu, J. Garcia, F. Ginés, E. Sánchez, V. Sanz, "Materias primas para la fabricación de suportes de baldosas cerámicas", $2^{\text {a }}$ Ed., Instituto de Tecnología Cerámica - ITC/AICE, Castañeda, Castellón, Espanha (2002) 292p.

[21] S. J. G. Sousa, J. N. F. Holanda, Ceram. Int. 31, 2 (2005) 215.

[22] A. P. F. Albers, F. G. Melchiades, R. Machado, J. B. Baldo, A. O. Boschi, Cerâmica 48, 305 (2002) 34.

[23] C. M. F. Vieira, J. N. F. de Holanda, D. G. Pinatti, Cerâmica 46, 297 (2000) 14.

[24] K. Okada, N. Õtsuka, J. Ossaka, J. Am. Ceram. Soc. 69,10 (1986) C251.

[25] A. Aras, Appl. Clay Sci. 24, 3-4 (2004) 257.

[26] K. Traoré, T. S. Kabré, P. Blanchart, Ceram. Int. 29, 4 (2003) 377.

[27] M. A. Qtaitat, I. N. Al-Trawneh, Spectrochim. Acta Part A 61 (2005) 1519.

[28] P. S. Nayak, B. K. Singh, Bull. Mater. Sci. 30, 3 (2007) 235.

[29] L. Maritan, L. Nodari, C. Mazzoli, A. Milano, U. Russo, Appl. Clay Sci. 31, 1-2 (2006) 1.

[30] E. V. Kalinkina, A. M. Kalinkin, W. Forsling, V. N. Makarov, Int. J. Miner. Process. 61, 4 (2001) 273.

(Rec. 18/10/2013, Rev. 05/02/2014, Ac. 15/02/2014) 